\title{
Methodological Issues in Conducting Treatment Trials for Psychological Nonepileptic Seizures
}

\author{
W. Curt LaFrance Jr., M.D., M.P.H. ${ }^{1,2}$, Andrew S. Blum, M.D., Ph.D. ${ }^{1}$, Ivan W. Miller, Ph.D. ${ }^{2}$, \\ Christine E. Ryan, Ph.D. ${ }^{2}$, and Gabor I. Keitner, M.D. ${ }^{2}$ \\ ${ }^{1}$ Department of Neurology and the Comprehensive Epilepsy Program, Brown Medical School, \\ Providence, Rhode Island ${ }^{2}$ Department of Psychiatry and Human Behavior, Brown Medical School, \\ Providence, Rhode Island
}

\begin{abstract}
A randomized, placebo-controlled trial has yet to be completed in patients with psychological nonepileptic seizures (NES). Treatment publications for NES are limited to class III trials and class IV reports. Little is written on the methodology of treatment trials in NES. The authors describe the procedures and limitations of such a trial to inform future NES treatment trials, based on their prospective, open-label pharmacological, feasibility trial. The authors review the recruitment, enrollment, completion of surveys, compliance, and follow-up of patients with NES. The majority of patients who enrolled, readily completed surveys and took the medication during the trial. Twelve patients were screened, eight enrolled, and six completed the trial. The authors discuss the use of outcomes and the various symptoms scales in the trial. A comprehensive neuropsychiatric initial assessment and assessing cognitive, emotional, behavioral, and psychosocial measures are important for monitoring the outcomes in NES treatment RCTs.
\end{abstract}

Patients with psychological nonepileptic seizures are often severely disabled, refractory to treatment, and frequently are encountered in neurology, psychiatry, and emergency departments. Nonepileptic seizures (NES), also referred to as pseudoseizures, are paroxysmal behaviors which have psychological comorbidities and are unresponsive to treatment with antiepileptic drugs. The phenomenology of NES is well delineated, including a preliminary understanding of risk factors and prognostic features. ${ }^{1}$ A number of studies exist on the diagnosis of NES, ictal semiology, comorbid psychiatric diagnoses, and neurological and neuropsychological characteristics of patients with NES. To date, no double-blinded, randomized, placebo-controlled trial has been completed for patients diagnosed with NES.

Given the paucity of systematic treatment trials for NES, much less is known about treatments of NES. A review of the literature reveals that only class III and IV studies are available, and no effective treatments have been developed. ${ }^{2}$ Patients with NES present unique challenges, given the overlap of neurological and psychiatric presentations. The number of papers in journals addressing clinical trial methodology in individual neurological or psychiatric disorders is limited. ${ }^{3-5}$ The few present do not adequately address when combined neuropsychiatric issues are present in a single trial. The purpose of this report is to describe

Address correspondence to Dr. LaFrance, Rhode Island Hospital, 593 Eddy Street, Potter 3, Neuropsychiatry, Providence, RI 02903; William_LaFrance_Jr@Brown.edu (e-mail).

Portions of this paper were presented at a poster session at the NINDS/American Neurological Association Symposium, in Toronto, Ontario, Canada, October 2, 2004. 
the open-label procedures and limitations of a feasibility trial in this specific neuropsychiatric population to aid and inform future NES treatment trials.

Patients with NES often have comorbid psychiatric conditions, such as major depressive disorder and anxiety disorders, including PTSD, or symptoms of depression, anxiety, and impulsivity seen in characterological disorders. ${ }^{6}$ Mood, anxiety, and impulsivity disorders are characterized by serotonin system dysregulation, and have been shown to be responsive to serotonergic medications. ${ }^{7}$ Clinical observation suggests that as some patients' depression and anxiety improve, their NES decrease.

Treatment strategies, in general, include primary prevention, risk factor reduction, and prophylactic and symptomatic interventions. Because symptomatic treatment for NES does not exist, one treatment strategy for patients is to treat the high frequency of comorbid psychiatric conditions that accompany NES. ${ }^{8}$ Reducing other medical illnesses or risk factors associated with stroke (e.g., diabetes, hypertension, and cholesterol), reduces the frequency of stroke. The pathophysiology of NES is less clear. A study ${ }^{9}$ assessing risk factors for persistent NES revealed that patients with unremitting NES had a markedly higher frequency of recurrent major depression and personality disorders, and a history of chronic abuse. ${ }^{9}$ Therefore, by analogy, we hypothesize that treating the most frequently occurring comorbid conditions associated with NES will reduce NES. Serotonin-modulating drugs, specifically selective serotonin reuptake inhibitors (SSRIs), are a reasonable pharmacological choice to safely treat these comorbid conditions. Of the SSRIs, sertraline has the broadest FDA indications and the least drug-drug interactions with other medications, a concern raised since many patients treated for seizures, whether epileptic or nonepileptic, are on antiepileptic drugs (AEDs).

We designed a treatment that targets depression, anxiety, and impulsivity, the comorbid diagnoses that most frequently accompany NES and are risk factors for NES persistence. ${ }^{9} \mathrm{We}$ intentionally focused on these disorders where the pathophysiology may be more responsive to pharmacological intervention. This approach may expand treatment options for patients with NES and also may ultimately build our understanding of the disorder itself. To prepare for a pharmacological double-blinded, randomized controlled trial for comorbid symptoms of NES, we conducted a prospective, open-label, feasibility trial. We describe methodological issues that arose in the conduct of the open-label trial.

\section{METHOD}

The protocol was approved by the Rhode Island Hospital (RIH) Institutional Review Board. Patients were referred to the RIH neuropsychiatry clinic after being diagnosed by capturing at least one of the patient's typical NES on vEEG in the RIH comprehensive epilepsy center between July 2002 to August 2003. The diagnosis of NES was defined as consisting of stereotypic, motoric manifestations, with or without change in level of consciousness, on vEEG with no epileptiform activity immediately before, during, or after the event. Patients who were potential study candidates underwent neuropsychiatric examination and clinical screening for major depressive disorder, anxiety disorders (generalized anxiety disorder, panic disorder, or posttraumatic stress disorder), or borderline personality disorder (or a combination of the diagnoses) by a board-certified neurologist and psychiatrist. For this SSRI treatment trial, we chose the most commonly occurring disorders in NES (depression, anxiety, and impulsivity) that have been shown to respond to serotonergic treatments.

Participants who met inclusion/exclusion criteria, between the ages of 18 to 65 years, provided written informed consent, and enrolled in the study. We restricted the age range to 18 to 65 years to minimize the potential age effect modification. It is well documented that NES occurs in children and that children tend to have better outcomes than adults with NES. ${ }^{10}$ Case reports 
and small series also show that NES occur in the elderly. ${ }^{11-14}$ Studies reporting outcome in the elderly with NES, however, have not been published.

Participants were administered the Structured Clinical Interview for DSM-IV Axis I Disorders (SCID) and Structured Interview for DSM-IV Personality Disorders (SID-P) by trained research interviewers. Inclusion criteria were vEEG diagnosis of NES and the presence of one of the aforementioned axis I diagnoses. Those with equivocal diagnoses on vEEG, such as simple partial-type NES, were excluded. Patients with mixed epileptic seizures and NES who could clearly distinguish between their events were included. One patient enrolled who clearly experienced both epileptic and nonepileptic seizures and could distinguish between the two. Other exclusion criteria included the presence of a current psychosis (hallucinations or delusions), current suicidality (plan and intent of killing oneself), current substance dependence (DSM-IV criteria), mental retardation, pending litigation, or disability application. Participants who were currently taking optimal doses of an antidepressant were excluded rather than washed out for this pilot trial with the reasoning that if they had NES on an optimized antidepressant, switching to another may affect the outcome measures. (Our optimized dose chart ${ }^{15,16}$ is available upon request). Patients on suboptimized antidepressants were eligible. Other pharmacological exclusions included taking any monoamine oxidase inhibitor, the triptans, and pimozide.

After enrolling, patients completed preenrollment seizure frequency, psychosocial functioning, and symptom scales. Historical and medical data were collected from chart review, patient query (open-ended and item-focused questions), and self-report surveys (Table 1). The seizure frequency in the 2 weeks prior to enrolling was obtained retrospectively to compare it to the initial baseline, a 2-week period of being untreated. The baseline period was designated as the time from enrollment (day 0) to initiation of medication (day 14).

Following the 2-week untreated baseline period, patients were given sertraline orally for 8 weeks, titrated from $25 \mathrm{mg}$ to $200 \mathrm{mg}$, once daily, as tolerated. (Patients with depression and anxiety respond to 50 to $200 \mathrm{mg}$ daily of sertraline. In light of the wide effective dose range of sertraline, the open-label trial protocol was flexible dose, titrated to $200 \mathrm{mg}$ or to maximum tolerable side effect while utilizing lower doses). Patients recorded their events, daily, on a seizure calendar. Collateral information from family informants was allowed and encouraged, given that some patients with epileptic and nonepileptic seizures are not aware of their events. Self-report and clinician evaluation symptom scales were administered biweekly during the medication dosing visits, with patients focusing on their symptoms for the 2 prior weeks. Total scores of these scales and calendars were used for data collection.

At week 10, patients in consultation with the study physician remained on sertraline or tapered off of the medication over the final 2 weeks of the treatment trial. If patients felt symptomatic improvement, they could continue on sertraline by prescription at their own cost.

After exiting the pharmacological trial, a questionnaire was administered via phone call followup by a trained researcher at months 4,8 , and 12 from date of enrollment to inquire about the persistence of seizures, current treatments, and psychosocial information.

\section{RESULTS}

We summarize the conduct of the study to address the methodological issues. Of 12 patients screened, nine participants were eligible and eight enrolled in the study. Three patients were excluded because of 1) their inability to differentiate between seizure types with mixed epilepsy and NES, and 2) being on optimized SSRI. One patient with driving restrictions did not enroll because of limited transportation. Of the eight enrollees, two patients dropped out of the study the day after they enrolled, one because he changed his mind and did not want to take sertraline, 
and the other because she could not complete the questionnaires with her dominant-handed weakness.

Of the nine patients eligible for the trial, eight enrolled, reflecting good recruitment feasibility for the study, with the readiness of physicians to refer patients with NES. The patients readily underwent screening and neuropsychiatric examination. Compliance with the protocol was good in five of the six participants. One participant did not attend the final visit but did report her symptom counts via telephone follow-up. Two of the participants required significant family promptings to keep follow-up visits. Family members drove participants to all of the visits. Study battery completion ranged from 40 to 80 minutes per visit. Patients with head injuries took longer to complete surveys.

Patients were able to report their events accurately on the prospective calendars during the pilot trial. Seizures were reported by the patients and their family members as the number of NES occurring at each of the 2 week follow-up visits. The majority of patients were able to record their own events. One patient was reminded by his family member of the number of events he had. Seizure frequencies in the 2 weeks prior to enrollment and in the baseline 2 weeks after enrollment (prior to receiving sertraline), were similar. We found that two patients, who had a frequency of 10 or more seizures every 2 weeks, had a worsening of their NES or no change. Those with less than 10 seizures every 2 weeks had a reduction in the frequency of NES.

We found the use of multiple symptom scales as secondary outcome measures to be informative. We collected neurological, psychiatric, psychosocial, and quality of life measures on six of the eight patients. The outcomes are summarized in Table 2, reporting median and range scores (the appropriate statistical summary for small sample size). We have intentionally not performed statistical t tests or chi square pre- and posttreatment comparisons, because this open-label study was a feasibility trial for a randomized controlled trial only and was not powered for such comparisons. Thus, it is inappropriate to perform those tests with these openlabel data.

The baseline values of the patients reveals that they were symptomatic across all administered psychological and social measures. For a reference point, the cutoff scores found in healthy control populations or in asymptomatic populations for each of the scales are listed in brackets. On average, these patients with NES had moderate to severe depression scores (Hamilton, $<7$, and Beck Depression Scales, <14), symptoms related to trauma (Davidson Trauma Scale, <17), moderate to severe impulsivity (Barrett Impulsivity Scale, <70), dissociative experiences (Dissociative Experiences Scale, <5), elevated somatic scores (Symptom Checklist 90, <120), impaired social functioning (Global Assessment of Functioning, >80, and LIFE-RIFT, <9 [in recovery]), family dysfunction (Family Assessment Device, FAD General Functioning subscale $<2.0]$, and maladaptive coping patterns (Ways of Coping).

\section{DISCUSSION}

We report on an open-label feasibility trial of sertraline for comorbid psychiatric disorders in eight patients with NES. Patients with NES are symptomatic across a number of neurological, psychological, and social variables. Our evaluations showed the presence of pathological scores on numerous scales revealing that these patients have a variety of psychosocial issues, along with their events. These findings are similar to what van Merode et al. ${ }^{17}$ showed in their study on psychopathological symptoms in patients with NES. Baseline scores were in the symptomatic range, indicating a need for looking at a broad set of measures for primary and secondary outcomes. Depression and anxiety scores were in the clinical range, validating our focus on the commonly occurring comorbidities for treatment. Interestingly, DES scores were consistent with a PTSD population, and not those with dissociative identity disorders. ${ }^{18}$ 
Patients with NES and comorbid psychiatric symptoms were able to complete the surveys and tolerate flexible dose sertraline over the course of the trial.

Patients enrolling in a treatment trial receive more attention than they typically receive in routine medical encounters. This may be particularly true for patients diagnosed with NES. Giving focused attention to patients who are often seen by a number of providers may have an impact on the frequency of events. In statements with mixed relief and exasperation, a number of patients with NES reported appreciation that "at least now somebody is trying to help with this." The Hawthorne effect (the positive effect observed in patients from the attention received in a study) may come into play with trials enrolling these patients. ${ }^{19} \mathrm{We}$ collected the retrospective 2 week seizure count at enrollment, and to address the potential Hawthorne effect, compared it to the frequency of events in the 2 week treatment free baseline period ( 8.4 and $7.5 \mathrm{NES}$ ). Monitoring patients for seizures and symptoms prior to receiving the intervention may be essential to look for changes, either positive or negative, from enrollment to initiation of therapy. The Hawthorne effect may be due to the researcher's cumulative attention over time, so the baseline comparison may not mitigate the effect. The potential confound underscores the need for a placebo-controlled trial. A blinded baseline also may help to establish what effect trial enrollment has on other symptoms.

For statistical analysis, dichotomous groups based on symptom severity may need to be established. In our trial, patients with a baseline frequency of 10 or more events every 2 weeks showed either worsening or no improvement in their seizure frequency. The 10 events every 2 weeks was an ad hoc finding; there was no preset cutoff for severity related to seizure frequency. There is a large variance in the number of events that different patients with NES experience. This finding raises the possibility that a high baseline frequency may yield worse outcomes and may need to be addressed when designing treatment trials. Stratifying the subjects into high and low seizure frequencies may be helpful to better evaluate the impact of an intervention but may influence the statistical analysis. Analogously, patients with higher psychiatric symptom scores or psychosocial dysfunction may experience worse outcomes. The effect of stratification on sample size calculations would need to be explored in more detail for the randomized controlled trial, and large numbers of patients may be needed to address these potential stratification issues.

It will be important to examine both short- and long-term outcomes in NES trials to monitor for a sustained treatment effect. After vEEG diagnosis of NES, patients may have an immediate reduction in the number of events, ${ }^{20}$ however, this effect is not longstanding in the majority of patients. ${ }^{21}$ Since it is unclear why a temporary NES reduction occurs immediately following diagnosis, it will be important to follow patients after any intervention to assess its true benefit. We are following the subjects in this open-label trial with phone calls 12 months after enrollment to assess seizure status, medication usage, global functioning, and for treatment dropout rates. The results of the 12-month follow-up phase of the study are not yet complete.

Factors affecting our recruitment retention included our inclusion/exclusion criteria and participant dropouts. We discussed the rationale for the age restrictions above. To limit age effect bias, future trials may either focus on a population younger than 18 years or may include patients older than age 65 . The age restriction should not be overly limiting to recruitment. In general, clinical trials report retention rates of $10 \%$ to $15 \%$, from screen to completion. ${ }^{22} \mathrm{We}$ obtained a $50 \%$ retention rate, and this argues for the success of future NES trials.

We found limitations in conducting treatment trials in NES in physical, social, logistic, and recruitment realms (Table 3). The typical problems with recruitment and with retaining patients seen in neurological clinical trials or in psychiatric clinical trials are compounded in a trial with combined neuropsychiatric issues present. For example, depression trials typically do not have 
to deal with driving restrictions, and epilepsy trials do not typically have to monitor for suicidality. In the absence of articles that address patient difficulties and complexities together, we describe issues that presented in a NES population. These problems also may arise in treatment trials with other neuropsychiatrically ill populations, such as traumatic brain injury.

Crises, lability, and approach/avoidance patterns present significant challenges to recruitment and retention in this population. A number of patients with NES present in crisis and with frequent events. When assistance is offered, however, they reject support. Two patients dropped out of the open-label trial the day after enrolling. One patient who was admitted to the inpatient unit for an acute exacerbation of depression dropped out after reviewing the battery of questionnaires and did not want to switch from suboptimized paroxetine to sertraline. The other dropout was an inpatient in the epilepsy monitoring unit who experienced physical limitations that included developing dominant-handed hemiparesis following some of her NES.

Cognitive and physical limitations in NES patients that may be neurologically based or that may be part of other somatoform symptoms may affect participation in trials. In order to obtain a comprehensive assessment, patients completed a large battery of self-report questionnaires measuring the frequency of NES, depression, anxiety, dissociative, somatoform, and traumaassociated symptoms, family functioning, quality of life, and other psychosocial variables. A number of patients with NES have neurological deficits 9 and a high frequency of nonseizure conversion disorders. ${ }^{6}$ If dominant hemiparesis, either of physiological or of psychogenic etiology, exists, patients may be unable to participate fully in trials that document broad psychosocial factors through self-report batteries. Also, if patients are on numerous CNS active medications, cognitive slowing may be a limitation to timely completion of self-report batteries.

In our pilot trial, we elected to accept the patients with focal findings and cognitive slowing, and asked them to complete all the forms. This led to losing one participant and resulted in modifying our randomized controlled trial. Researchers accept a certain attrition rate in trials. When designing studies, it is important to realize that not including patients with brain damage will potentially affect generalizabilty to other NES populations. If, on the other hand, these patients are to be included, an abbreviated battery or clinician ratings may be the "least common denominator" used in the final outcomes comparisons among all subjects in trials.

A logistical issue that may limit participation in NES trials not present in psychiatric trials is driving. Driving restrictions in patients with epilepsy vary by state. ${ }^{23}$ The issue of restricting patients with NES driving is less clear. ${ }^{24}$ A survey ${ }^{25}$ of neurologists revealed that many physicians recommend restricting driving privileges in patients with NES. Benbadis et al. ${ }^{25}$ did not find a difference in the number of accidents in 20 NES patients when compared with general population accident rates. Driving, and the often lengthy distance to a research center, may be a major limitation for patients. Patients with NES also expressed reservations about using mass transit, for fear of having an event on public transportation. Enlisting family or friends, or budgeting for taxis for patient transportation may be one way to address this potential problem in prospective trials for NES.

Given our current lack of knowledge in NES treatments, including patients who have mixed epileptic and nonepileptic seizures may or may not be considered a limitation. Reports on comorbidity reveal that only $5 \%$ to $10 \%$ of patients have mixed seizures. ${ }^{26-28}$ To limit the possibility of inaccurate seizure frequency quantification, one could argue that the ideal treatment trial will select only patients with lone NES to utilize a more "pure" study population. Acknowledging the low prevalence of mixed seizures, for this feasibility trial, we elected to enroll these patients who could readily distinguish between their events. Trials with larger 
samples may opt for the "purist" approach of enrolling only lone NES patients, realizing that this may affect generalizability to the subset with mixed seizures.

One concern regarding the "ideal" NES population is the difficulty of diagnosing, enrolling, and then retaining this difficult-to-engage population. Including mixed patients may shed light on similarities and differences between the nonepileptic and epileptic seizures and mixed populations. Many patients with mixed seizures are often readily able to identify and distinguish their various events with a careful initial history. When asked about their seizure descriptions, patients with mixed seizures describe, for example, the seizures they experience at night with postictal body pain and incontinence. These are contrasted with the other events that occur during the day and are triggered by observing a traumatic event on the TV: "I'm usually okay in a few minutes right after that type of seizure." These distinctions are documented with the absence of epileptiform activity on vEEG during the daytime NES, and presence of epileptiform activity on the nocturnal generalized tonic clonic seizure, which helps to validate the distinction of the two seizure types. When monitoring outcomes, training the patient and the family to record these different events separately will be essential.

Based on our hypothesis testing the effect of a SSRI, we sought patients with NES who had no prior treatment with psychotropic medications. Another population "purity" methodological issue in the NES population is one of finding incident cases for psychotropic naïve patients. Studies show that there is a 7-year diagnostic delay between onset of seizures and diagnosis of NES. ${ }^{29}$ In the ensuing time, patients are treated with a number of antiepileptic drugs and also with psychotropic medications. ${ }^{30}$ Most of the patients in our study had prior exposure to antidepressants. We found, however, that the dosing may have been below optimal pharmacological level. It is unclear whether prior medication exposure limits potential response to an optimized drug. Also, from a clinical trial perspective, the effect of stopping a current medication may have as much of an impact on outcome as starting a new medication. Given that patients with NES have had anti-epileptic drug and psychotropic exposures, the decision to discontinue all psychotropic medications, or antiepileptic drugs, or to add the study medication to the current regimen, is one of considerable importance for future NES treatment trials. In our open-label trial, patients stopped their antidepressants prior to enrolling.

The means and timing of establishing the NES diagnosis are linked to enrollment procedures. We found a 12-month delay from vEEG diagnosis to obtaining treatment for NES. Once referred for the study, patients who were eligible were enrolled within 1 month. This diagnostic delay deserves mention because the time lag may affect prognosis. Poorer prognosis is seen in patients with a long history of NES. ${ }^{31}$ Patients who have had nonepileptic seizures for less than 12 months required fewer treatment sessions to reach cessation of events than those who had their events for more than a year. ${ }^{32}$ A number of factors could account for NES diagnosis to treatment delay. Patients may not accept the diagnosis, moving from a "neurological problem to a psychiatric one." 33 Referrals between neurologists and psychiatrists may be limited because of a lack of interdisciplinary discussion. ${ }^{34}$ The diagnosis is most often made at tertiary care centers with LTM units, and communication of the diagnosis to the referring physician may not occur until a report is generated. The delay from vEEG diagnosis to treatment could be shortened by addressing the above issues.

A common primary outcome in antiepileptic drug trials is number of seizures. In our trial, we found that patients scored at pathological or symptomatic levels on a number of other measures of psychosocial functioning. In nonepileptic or epileptic seizures, it is important for us to realize that treating the ictus is not the only goal of treating patients with seizures, and comorbidities affect outcomes. Treatment trials that consider various psychosocial issues in the lives of patients with NES may lead to improved treatment outcomes. 
In summary, prospective, controlled treatment trials in patients with NES are necessary to determine effective interventions. In demonstrating the feasibility of an open-label trial, we address the methodological issues to inform conducting rigorous randomized controlled treatment trials in NES. From our open-label feasibility trial, we learned that relevant factors for trials in NES patients include: NES and psychosocial symptom severity, age differences, personal physical characteristics, logistical restrictions, the presence of mixed seizures, prior antiepileptic drug/antidepressant treatments, and having a breadth of outcome measures. Being aware of these design and methodological issues may improve enrollment, retention, and outcomes, and facilitate future NES treatment trials and for other neuropsychiatric disorders.

\section{Acknowledgments}

This study is funded by Grant 1 K23 NS 45902-01 from the National Institute of Neurological Disorders and Stroke.

\section{References}

1. Barry JJ. Nonepileptic seizures: an overview. CNS Spectrums 2001;6:956-962. [PubMed: 15311188]

2. LaFrance WC Jr, Devinsky O. The treatment of nonepileptic seizures: historical perspectives and future directions. Epilepsia 2004;45(suppl2):15-21. [PubMed: 15186340]

3. Haythornthwaite JA. Clinical trials studying pharmacotherapy and psychological treatments alone and together. Neurology 2005;65(suppl 4):S20-31. [PubMed: 16385101]

4. Fawcett J, Epstein P, Fiester SJ, et al. Clinical management-imipramine/placebo administration manual: NIMH Treatment of Depression Collaborative Research Program. Psychopharmacol Bull 1987;23:309-324. [PubMed: 3303100]

5. Leppik IE, Brodie MJ, Saetre ER, et al. Outcomes research: clinical trials in the elderly. Epilepsy Res 2006;68S:S71-S76. [PubMed: 16413171]

6. Bowman ES, Markand ON. Psychodynamics and psychiatric diagnoses of pseudoseizure subjects. Am J Psychiatry 1996;153:57-63. [PubMed: 8540592]

7. Vaswani M, Linda FK, Ramesh S. Role of selective serotonin reuptake inhibitors in psychiatric disorders: a comprehensive review. Prog NeuroPsychopharmacol Biol Psychiatry 2003;27:85-102. [PubMed: 12551730]

8. LaFrance WC Jr, Devinsky O. Treatment of nonepileptic seizures. Epilepsy Behav 2002;3(5 suppl): 19-23. [PubMed: 12609316]

9. Kanner AM, Parra J, Frey M, et al. Psychiatric and neurologic predictors of psychogenic pseudoseizure outcome. Neurology 1999;53:933-938. [PubMed: 10496249]

10. Wyllie E, Friedman D, Luders H, et al. Outcome of psychogenic seizures in children and adolescents compared with adults. Neurology 1991;41:742-744. [PubMed: 2027493]

11. Fakhoury T, Abou-Khalil B, Newman K. Psychogenic seizures in old age: a case report. Epilepsia 1993;34:1049-1051. [PubMed: 8243356]

12. Shulman KI, Silver IL. Hysterical seizures as a manifestation of "depression" in old age. Can J Psychiatry 1985;30:278-280. [PubMed: 4016665]

13. Lempert T, Dieterich M, Huppert D, et al. Psychogenic disorders in neurology: frequency and clinical spectrum. Acta Neurol Scand 1990;82:335-340. [PubMed: 2281751]

14. Kellinghaus C, Loddenkemper T, Dinner DS, et al. Nonepileptic seizures of the elderly. J Neurol 2004;251:704-709. [PubMed: 15311346]

15. Keller MB. Undertreatment of major depression. Psychopharmacol Bull 1988;24:75-80. [PubMed: 3387524]

16. Oquendo MA, Baca-Garcia E, Kartachov A, et al. A computer algorithm for calculating the adequacy of antidepressant treatment in unipolar and bipolar depression. J Clin Psychiatry 2003;64:825-833. [PubMed: 12934985]

17. van Merode T, Twellaar M, Kotsopoulos IA, et al. Psychological characteristics of patients with newly developed psychogenic seizures. J Neurol Neurosurg Psychiatry 2004;75:1175-1177. [PubMed: 15258225] 
18. Bernstein EM, Putnam FW. Development, reliability, and validity of a dissociation scale. J Nerv Ment Dis 1986;174:727-735. [PubMed: 3783140]

19. West ED, Jackson A, Physentides A, et al. Randomized comparative trial of a ward discussion group. Br J Psychiatry 1982;141:76-80. [PubMed: 7052184]

20. Farias ST, Thieman C, Alsaadi TM. Psychogenic nonepileptic seizures: acute change in event frequency after presentation of the diagnosis. Epilepsy Behav 2003;4:424-429. [PubMed: 12899864]

21. Wilder C, Marquez AV, Farias ST, et al. (Abstract 2.469) Long-term follow-up study of patients with PNES. Epilepsia 2004;45(suppl7):349.

22. Keitner GI, Posternak MA, Ryan CE. How many subjects with major depressive disorder meet eligibility requirements of an anti-depressant efficacy trial? J Clin Psychiatry 2003;64:1091-1093. [PubMed: 14628985]

23. Krauss GL, Ampaw L, Krumholz A. Individual state driving restrictions for people with epilepsy in the US. Neurology 2001;57:1780-1785. [PubMed: 11723263]

24. Iriarte J, Parra J, Urrestarazu E, et al. Controversies in the diagnosis and management of psychogenic pseudoseizures. Epilepsy Behav 2003;4:354-359. [PubMed: 12791342]

25. Benbadis SR, Blustein JN, Sunstad L. Should patients with psychogenic nonepileptic seizures be allowed to drive? Epilepsia 2000;41:895-897. [PubMed: 10897163]

26. Lesser RP, Lueders H, Dinner DS. Evidence for epilepsy is rare in patients with psychogenic seizures. Neurology 1983;33:502-504. [PubMed: 6682199]

27. Benbadis SR, Agrawal V, Tatum IV. How many patients with psychogenic nonepileptic seizures also have epilepsy? Neurology 2001;57:915-917. [PubMed: 11552032]

28. Martin R, Burneo JG, Prasad A, et al. Frequency of epilepsy in patients with psychogenic seizures monitored by video-EEG. Neurology 2003;61:1791-1792. [PubMed: 14694050]

29. Reuber M, Fernandez G, Bauer J, et al. Diagnostic delay in psychogenic nonepileptic seizures. Neurology 2002;58:493-495. [PubMed: 11839862]

30. de Timary P, Fouchet P, Sylin M, et al. Nonepileptic seizures: delayed diagnosis in patients presenting with electroencephalographic (EEG) or clinical signs of epileptic seizures. Seizure 2002;11:193197. [PubMed: 12018963]

31. Lempert T, Schmidt D. Natural history and outcome of psychogenic seizures: a clinical study in 50 patients. J Neurol 1990;237:35-38. [PubMed: 2319265]

32. Rusch MD, Morris GL, Allen L, et al. Psychological treatment of nonepileptic events. Epilepsy Behav 2001;2:277-283. [PubMed: 12609370]

33. LaFrance WC. How many patients with psychogenic nonepileptic seizures also have epilepsy? Neurology 2002;58:990-991. [PubMed: 11914432]

34. Kanner AM. More controversies on the treatment of psychogenic pseudoseizures: an addendum. Epilepsy Behav 2003;4:360-364. [PubMed: 12791343] 
TABLE 1

Patient Medical History Obtained by Interview and Record Review $(\mathrm{N}=8)$

\begin{tabular}{|c|c|c|c|c|}
\hline & & (SD) & $\mathbf{N}$ & $(\%)$ \\
\hline \multicolumn{5}{|l|}{ Sociodemographic Factors ( $\mathrm{N}=8$ ) (self-reported) } \\
\hline Age (years) & 39.6 & $(11.6)$ & & \\
\hline Age of NES onset & 40.5 & $(12.3)$ & & \\
\hline Gender (female) & & & 6 & (75) \\
\hline Education (years) & 13.3 & $(2.69)$ & & \\
\hline Unemployed currently & & & 4 & $(50)$ \\
\hline Receiving disability currently & & & 4 & $(50)$ \\
\hline Married currently & & & 4 & $(50)$ \\
\hline Driving currently & & & 1 & (12) \\
\hline \multicolumn{5}{|l|}{ Clinical Diagnosis ${ }^{*}$ (made by M.D.) } \\
\hline Mood disorder & & & 8 & $(100)$ \\
\hline Anxiety disorder & & & 7 & $(87)$ \\
\hline Impulsivity (cluster B personality) & & & 2 & (25) \\
\hline Somatoform disorder (other than NES) & & & 1 & $(12)$ \\
\hline \multicolumn{5}{|l|}{ Clinical Factors (from history at baseline) } \\
\hline History of trauma/abuse & & & 4 & $(50)$ \\
\hline Previous psychotherapy & & & 4 & $(50)$ \\
\hline Treated with psychotropic medications (past and current) & & & 7 & (87) \\
\hline Benzodiazepines & & & 6 & (75) \\
\hline Antidepressants & & & 7 & (87) \\
\hline Antipsychotics & & & 2 & (25) \\
\hline On antiepileptic drugs (AEDs) at baseline & & & 7 & (87) \\
\hline Average total number of lifetime AEDs & 5.4 & $(4.2)$ & & \\
\hline Estimated number of seizures in 14 days pre-enrollment & 8.4 & $(14.3)$ & & \\
\hline Average time from NES diagnosis to NES treatment (months) & 12.4 & $(20.2)$ & & \\
\hline Abnormal neurological exam at enrollment & & & 6 & (75) \\
\hline Abnormal MRI of the brain (past or at enrollment) & & & 5 & $(62)$ \\
\hline \multicolumn{5}{|l|}{30 minute tracing EEG: } \\
\hline Interictal epileptiform activity & & & 4 & $(50)$ \\
\hline Slowing only abnormality & & & 1 & (12) \\
\hline Biological family history of seizures & & & 1 & (12) \\
\hline History of head injury & & & 1 & (12) \\
\hline
\end{tabular}

* Not mutually exclusive 
TABLE 2

Example of Types of Assessment Ratings at Baseline and Completion $(\mathrm{N}=6)$

\begin{tabular}{lcccc}
\hline & \multicolumn{2}{c}{ Baseline (enrollment) } & Completion (week 10) \\
\cline { 2 - 5 } Scale [cutoff] & Median & Range & Median & Range \\
\hline Modified Hamilton & 25 & 13 to 28 & 17 & 7 to 35 \\
$\begin{array}{l}\text { Depression Scale [<7] } \\
\text { Beck Depression Inventory - II }\end{array}$ & 26 & 10 to 44 & 11 & 2.0 to 36 \\
[<14] & 26.0 & 7 to 118 & 22.5 & 6 to 78 \\
$\begin{array}{l}\text { Davidson Trauma Scale [<17] } \\
\text { Barrett Impulsivity Scale [<70] }\end{array}$ & 80 & 51 to 93 & 68 & 54 to 83 \\
Dissociative Experiences & 31.8 & 11 to 44 & 17.3 & 11 to 176 \\
$\begin{array}{l}\text { Scale [<5] } \\
\text { Symptom Checklist 90 [<120] }\end{array}$ & 148 & 42 to 201 & 110 & 25 to 85 \\
Global Assessment of & 49 & 40 to 60 & 55 & 1.00 to 2.83 \\
$\begin{array}{l}\text { Functioning* [>80] } \\
\text { Family Assessment Device: }\end{array}$ & 2.00 & 1.33 to 2.58 & 1.79 & 6 to 30 \\
General Functioning Subscale & & & 15 & 0 to 64 \\
Score [<2.00] & & 7 to 28 & 2 & \\
LIFE-RIFT (QoL measure) & 20 & 1 to 34 & & \\
[<9] frequency during trial & 7.5 & & & \\
NES freekly sum) & & & & \\
\hline
\end{tabular}

For all assessments except *, a higher score indicates a worse condition.

Cutoff scores in patients and healthy subjects are presented in brackets by the scale.

( $\mathrm{p}$ values were intentionally not analyzed in this feasibility open-label trial, as it is not powered for such calculations) 
TABLE 3

NES Treatment Trial Issues and Obstacles

- $\quad$ NES severity/frequency

- Psychosocial symptom severity

- $\quad$ Outcome differences in younger vs. older cohorts

- Personal physical characteristics-hemiparesis and cognitive symptoms

- Logistical restrictions-transportation/driving

- Distinguishing mixed nonepileptic/epileptic seizures

- Prior antiepileptic drug/antidepressant treatments

- Choice of primary outcome measures 